\title{
Analyse the method of scientific teaching in 'Digital circuit'
}

\author{
Fengyuan Zhu' ${ }^{1}$, Yi Deng ${ }^{1, *}$ \\ ${ }^{1}$ Electron information Engineering College of Hankou University, Wuhan China \\ *402345008@qq.com
}

\begin{abstract}
Digital circuit' as the backbone courses of electronic information engineering has the practical applicability brightly. It plays an important role to student's professional technical ability training. Based on the 'Digital circuit' course characteristics, and combine with the author's experience, who engaged in the course teaching a lot of years. This article analyzes the 'Digital circuit' course teaching method innovation from the theoretical teaching, practical teaching and curriculum design.
\end{abstract}

Keywords: Digital circuit; Teaching method; Innovation

\section{Introduction}

As the core courses in electronics professional, Digital circuit plays an important role in the development of the world, which is also widely used in daily life. Through this course, students are enabled to lay the solid foundation for the follow-up professional courses by getting hold of the basic knowledge of digital circuit, basic analysis method and the application of basic skills, as well as to learn to use the standard integrated circuits and programmable logic devices, and qualified the ability in accordance with the requirements of practical application of these units and components to a simple digital electronic system.

Making technology of application-specific integrated circuit (ASIC) ${ }^{[1]}$ has been becoming mature, while integration and wiring accuracy is higher more judging from development of digital technology. And with the development of computer technology, we can use hardware description language and electronic design automation (EDA) software to implement the digital circuit or analysis, simulation and design of digital system.

College teaching work must be adapted to international and domestic level of digital electronic technology development, the traditional teaching methods need to be improved in creative thinking and practical ability with the current stage of development, so as to cultivate excellent applicable talents.

\section{The present status of 'Digital circuit' education}

The basic principle, and knowledge of digital electronic technology will be mastered by the students, as well as experiment skills and logical thinking ability by learning the digital circuit course, in order to accumulate knowledge for the follow-up professional course especially hardware related. That can cultivate ability consulting manual and other reference books, commonly used digital integrated device parameters, and the practical problems encountered in the process of practice. And act as the necessary foundation for the practical work.

The requirement of talent cultivation must be keeping abreast of the times, with the rapid development of integrated electronic and computer technology, while the essence of education is to cultivate high-quality talents adapting to the development of society, so comprehensive education must follow the pulse of times with constant innovation. At present, most traditional digital circuit teaching still rest on theory rather than practice, we should make the teaching focus on improving practice ability follow the principle of the teaching reform, while the informazation socialization has been becoming increasingly higher, so as to adapt social development and cultivate innovative talents with high quality. 


\section{Primary analysis of teaching reform}

The teaching module of digital circuit consists of three parts: theory teaching, practice teaching, and curriculum design. With the purpose to ensure the integrity and effectiveness of teaching in the three stages, from basic knowledge to hands-on practice and ability.

\subsection{Theory teaching}

First of all, theory teaching should be combined with the current status of the development of digital electronic technology, while the traditional theory knowledge still mostly stays in small and medium scale integrated circuit, however the integrated circuit can be consisted of billions of transistors and wiring width around submicron magnitude, while development of digital technology is quite fast. As chips and wiring, the chips has decreased in power consumption but increased in speeds. Secondly, great attention must be paid to highlight the difficult knowledge and sort out the main research object of digital circuit being logic of the relationship between input and output, logic algebra is adopted as analysis tools in digital circuit different from the analog circuit as the foundation of learning. Digital circuit consist of the two major: combinational logic circuit and sequential logic circuit, it is important to grasp the analysis and design method of these two types circuit. At the same time, there should place emphasis on selecting textbook based on basic knowledge. With my experience as an example, the textbook is "digital electronic technology" edited by Yi Deng and Liping Wang, the first three chapters act as the basic content: numeral system and code, logic algebra foundation, integrated logic gate, the middle two chapters act as the full text of focus: combinational logic circuit and sequential logic circuit, after three chapters act as mainly application. Thirdly, reinforce element characteristic analysis by teaching, the basic logic devices of digital circuit consist of integration logic gate, as the basic elements, MOS tubes and transistors in digital circuit features similar to switch.

\subsection{Practical teaching}

As a practical course, Digital circuit requires pay attention to the emphasis on students' practical operating ability ${ }^{[2]}$,practice teaching serve as the key part of this course, it would seize rationality, scientific nature and validity. Through the experimental operation,students can consolidate the knowledge, as well as combine abstract theory with real components and products, in order to obtain intuitive for practical application of digital electronic technology. By practical teaching, students can be able to prepare for experiment via reading material or material, use commonly instruments with the help of textbooks or instrument instructions, use theoretical knowledge to make preliminary analysis of phenomenon, record and handle experimental data, draw curves, illustrate experimental results, and write qualified reports, as all as complete simple design experiments. It suggest arranging many verification and design experiment few demonstration and comprehensive experiment in the practice course.

\subsection{Curriculum design}

Curriculum design act as comprehensive test for learning outcomes ${ }^{[3]}$, which not only to examines proficiency of curriculum knowledge but to the promotion of core knowledge. According to the author's experience in teaching, curriculum design should be related to the practical application of digital circuit and combined with the current trend of electronic technology development, which targeted to the arrangement of curriculum design in order to apply it based on electronic design automation software.

\section{Integrate EDA software platform into classroom teaching}

With the development of computer technology, analysis, simulation and design of digital circuit or system can be realized by hardware description language and EDA software ${ }^{[4]}$ with the help of computer automatically. EDA software act as computer software system developed on the basis of electronic CAD technology, that commonly used in electronic circuit design and simulation software include: Protues, Protel, PSPICE software, EWB, etc., which can be powerful circuit design and simulation software. It is an essential skill for electronic majors to master EDA technology, and it is 
appropriate to combine the teaching content with the EDA platform in the course of teaching. Here is an example of EDA converter using ${ }^{[5]}$ in teaching.

D/A converter (DAC) converts digital signals to analog signals, DAC0832 chip is an 8-bit integration DA converter. A waveform generator can be maded with the DAC0832 directly connected with 51 series microcontroller.

The simulation circuit shown in Fig.1 is designed by proteus, and waveform generated via the appendix 1 program can be watched on the oscilloscope.

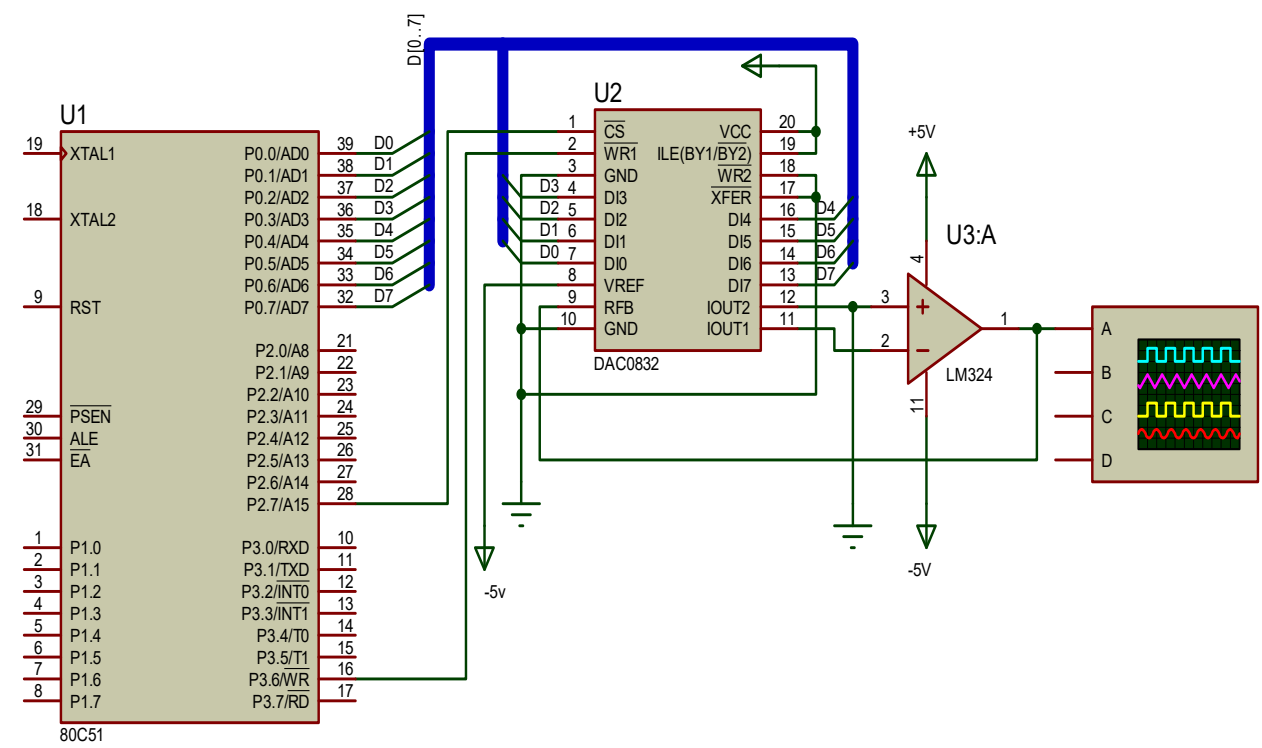

Fig.1. Emulated circuit diagram

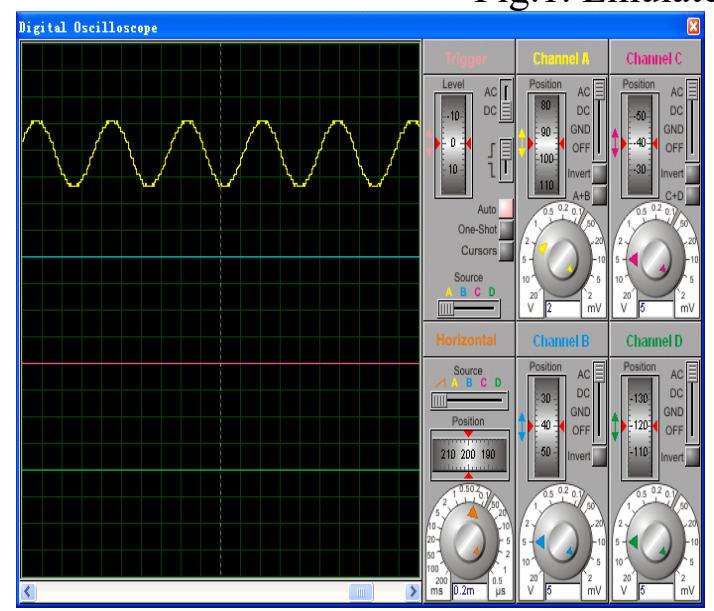

(a) Sine wave

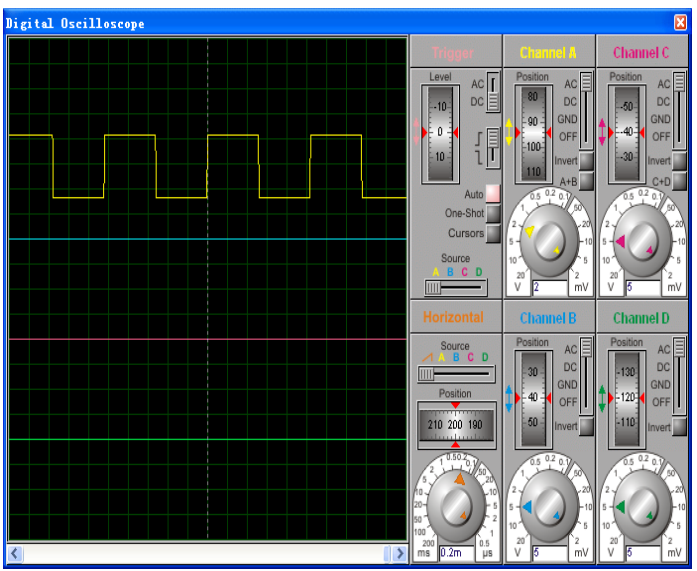

(c) Square wave

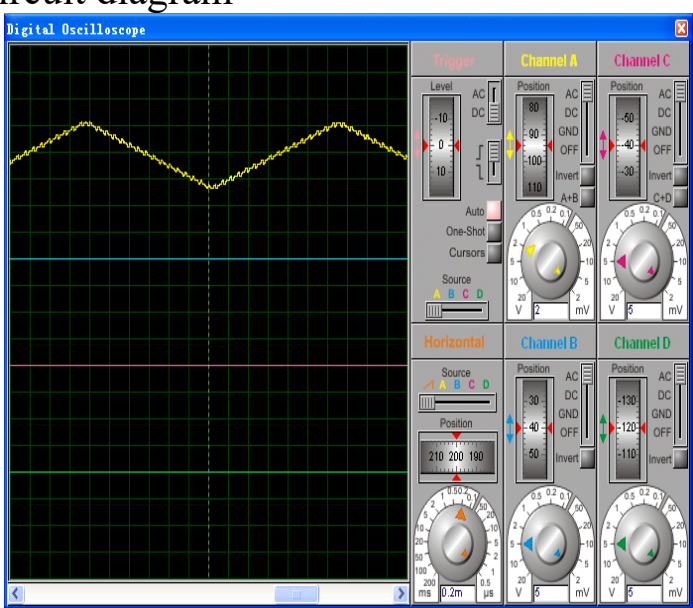

(b) Triangle wave

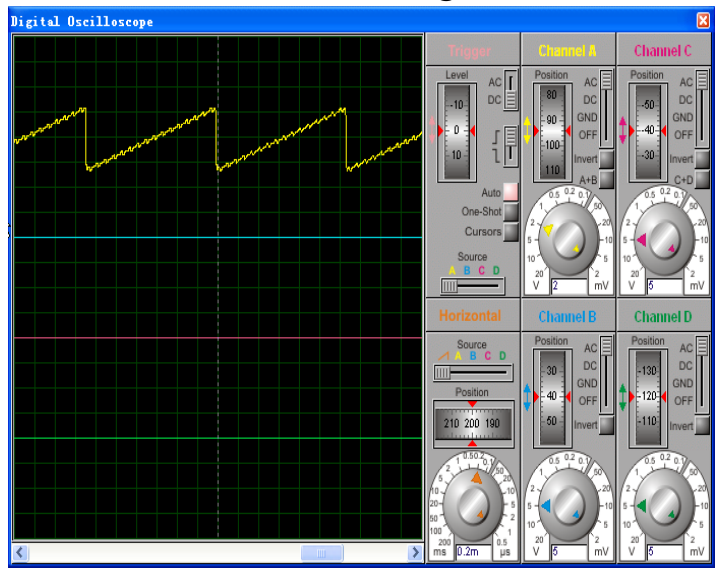

(d) Sawtooth wave

Fig.2. Various waveform schematics 
From the graph, that can clearly observe sine wave(Fig.2(a)), triangle wave(Fig.2(b)), square wave(Fig.2(c)) and sawtooth wave(Fig.2(d)) by using proteus software on D/A converters 0832 application of the simulation.

\section{Conclusions}

Digital circuit teaching present situation and the existing problems have been analyzed in this paper with the background of digital technology development and digital circuit teaching. Strategies and suggestions have been put forward by relevant combined with the author's teaching experience, which played a positive role in the new era of curriculum reform.

\section{References}

[1] Yuanchao Liu. The reform of digital circuit teaching and research [J],journal of the Yangtze University journal (natural science), September 6, 2009.

[2] Fu Li, Jing Dong, Yuanong Zhang, Xin Gong . Digital circuit foundation teaching reform research [J], digital communication, 2012.06.

[3] Wei Song,Youlian Zhu."digital circuit" curriculum design teaching reform exploration [J], journal of JiangSu Technical Teachers College, 2011.08.

[4] Zhengwei Zhu .EDA technology and application [M]. Beijing: Tsinghua University press, 2005.

[5] Yi Deng, Liping Wang. Digital electronic technology [M], Wuhan: Huazhong University of Science and Technology Press,2015.

\section{Author introduction:}

Fengyuan Zhu, female, born in October 1988, assistant professor of electronic information engineering at Hankou College, research direction: wireless communication, signal processing.

Yi Deng, female, born in April 1985, associate professor, dean of electronic information engineering college, Hankou College, research direction: intelligent control, digital image processing. 